\title{
CATÁLOGO DE LAS ALGAS VERDES COCALES DE LAS AGUAS CONTINENTALES DE ANDALUCÍA
}

\author{
Ingrid FANÉS TREVIÑO ${ }^{1 *}$, Augusto COMAS GONZÁLEZ ${ }^{2}$ \\ y Pedro M. SÁNCHEZ CASTILLO ${ }^{1}$ \\ ${ }^{1}$ Departamento de Botánica. Facultad de Ciencias. \\ Universidad de Granada. Avda. Fuentenueva s/n. 18071 Granada, España. \\ ${ }^{2}$ Centro de Estudios Ambientales de Cienfuegos, CITMA, \\ Calle 17 esq. Avda. 16, Reparto Reina, Cienfuegos, Cuba. \\ *Autor para correspondencia: ingrid@ugr.es
}

Recibido el 1 de junio de 2009, aceptado para su publicación el 17 de junio de 2009 Publicado "on line" en junio de 2009

\begin{abstract}
RESUMEN. Catálogo de las algas verdes cocales de las aguas continentales de Andalucía. En España los estudios realizados de los ecosistemas acuáticos continentales han tenido sobre todo un enfoque ecológico. En la mayoría de los trabajos limnológicos las algas se han considerado como productores primarios en su conjunto, pero en general no se ha profundizado en la identificación de estos organismos a niveles más concretos. En Andalucía se han realizado estudios taxonómicos de grupos como las diatomeas, pero son muy pocos los trabajos que incluyen consideraciones taxonómicas de especies de algas verdes cocales. Concretamente se han estudiado algunas especies presentes en embalses de Huelva y Sevilla, algunas lagunas de Sierra Nevada y en lagunas de las provincias de Almería, Málaga y Granada, e incluso en ambientes acuáticos urbanos, pero siempre bajo un punto de vista principalmente ecológico. En este trabajo, se presenta un catálogo que reúne todas las especies de algas verdes cocales citadas en estudios previos de aguas continentales de Andalucía, junto a nuevas citas procedentes de muestras recolectadas durante los años 2004 a 2006. Se incluye un total de 143 especies, de las cuales 9 se citan por primera vez en la Península Ibérica (Botryococcus terribilis, Chlorella minutissima, Desmodesmus grahneisii, Dicellula geminata, Dichotomococcus curvatus, Dictyosphaerium elongatum, Franceia ovalis, Paradoxia multiseta, Tetrachlorella ornata), 3 son nuevas citas para España (Kirchneriella rotunda, Crucigenia mucronata, Treubaria schmidlei), y 27 lo son para Andalucía. Se indican las provincias y localidades en que ha sido encontrado cada uno de los taxa, así como las referencias de las citas anteriores a este estudio.
\end{abstract}

Palabras clave. Flora, Catálogo, Algas verdes, Chlorococcales, Andalucía.

ABSTRACT. Catalogue of coccal green algae from continental waters of Andalusia. In Spain studies on continental aquatic ecosystems have had mainly an ecological approach. In most limnological papers algae are considered as primary producers as a whole, but in general there is no deepening into the identification of these organisms to more concrete levels. In Andalusia taxonomical groups such as diatoms have been studied,

Este trabajo ha sido parcialmente financiado por una beca del Programa de Formación de Profesorado Universitario del Ministerio de Educación y Ciencia a I. Fanés, por el Proyecto de Investigación Flora Ficológica de Andalucía de la Consejería de Medio Ambiente de la Junta de Andalucía y por el Proyecto CICYT REN 2002-04397-C03-01/GLO. 
but very few papers include taxonomical considerations on species of coccal green algae. Specifically, some species have been studied in reservoirs of Huelva and Seville, in some lakes of Sierra Nevada and in ponds in the provinces of Almeria, Malaga and Granada, and even in urban aquatic environments, but always under an ecological point of view mainly. In this paper we present a catalogue which includes all species of coccal green algae cited in previous studies of continental waters from Andalusia, together with new records from samples collected during the years 2004 to 2006. The catalogue includes a total of 143 species, of whom 9 are recorded for the first time in the Iberian Peninsula (Botryococcus terribilis, Chlorella minutissima, Desmodesmus grahneisii, Dicellula geminata, Dichotomococcus curvatus, Dictyosphaerium elongatum, Franceia ovalis, Paradoxia multiseta, Tetrachlorella ornata), 3 are new records for Spain (Kirchneriella rotunda, Crucigenia mucronata, Treubaria schmidlei), and 27 for Andalusia. We indicate the provinces and localities where each taxon has been found, as well as the references of the records previous to this study.

Key words. Flora, Catalogue, Green algae, Chlorococcales, Andalusia.

\section{INTRODUCCIÓN}

La mayoría de los estudios sobre algas realizados en los ecosistemas acuáticos continentales en España durante los últimos años han tenido sobre todo un enfoque ecológico. Dichos estudios han proporcionado catálogos más o menos completos, pero sin centrarse en consideraciones taxonómiconomenclarturales que permitieran avanzar en el conocimiento de la complejidad taxonómica de estos organismos, a la vez que facilitaran la actualización nomenclatural de sus especies según las últimas tendencias taxonómicas.

Sin embargo, una buena parte de estos catálogos "limnológicos" proporcionan una importante fuente para el conocimiento de las especies más abundantes de algas verdes cocales en el conjunto de los embalses españoles (Margalef et al., 1976, Sabater \& Nolla, 1991), y en algunas lagunas de distinto nivel trófico (Rojo, 1990; Romo, 1991; Hoyos, 1996; Negro, 2005). Otros trabajos más recientemente publicados (Pérez Baliero et al., 2002; Comas et al., 2006; Fanés Treviño et al., 2009) sobre las algas verdes cocales sí presentan un enfoque más taxonómico. La mayoría de citas procedentes del conjunto de estos trabajos han sido recopiladas hasta 1998 por Cambra y colaboradores.

En Andalucía se encuentra más avanzada la catalogación de otros grupos taxonómicos como las diatomeas, tanto en agua dulce (Linares Cuesta, 2003) como marina (Rivera González, 2004), pero son muy pocos los trabajos que incluyen consideraciones taxonómicas de especies de algas verdes cocales. Concretamente se han estudiado algunas especies presentes en embalses de Huelva y Sevilla (Toja, 1976, 1980; Toja et al., 1983), algunas lagunas de Sierra Nevada (Sánchez Castillo, 1986) y en lagunas de las provincias de Almería (Sánchez Castillo, 1982, 1987a), Málaga y Granada (de la Rosa, 1992), e incluso en ambientes acuáticos urbanos (Sánchez Castillo, 1983; Bolívar Galiano, 1994; Peraza Zurita et al., 2002).

Los datos aportados en este trabajo proceden, al menos en parte, del Proyecto Flora Ficológica de Andalucía, que ha pretendido iniciar el estudio y catalogación ficológica de la región, y del primer proyecto de Flora Ibérica de las Algas Continentales, que pretende elaborar un catálogo de todas las especies de algas presentes en los sistemas acuáticos continentales de la Península Ibérica, y del que ya se ha publicado el primer volumen (Cirujano et al., 2008). El presente trabajo trata de englobar los resultados de ambos proyectos, iniciando el estudio florístico del grupo de las algas verdes cocales en Andalucía (Fanés Treviño, 2008). Se muestrearon a lo largo de los años 2004 a 2006 lagunas, balsas y embalses de gran parte del territorio andaluz. La mayoría de las lagunas muestreadas están situadas en 


\begin{tabular}{|c|c|c|c|c|}
\hline Localidad & Provincia & $\begin{array}{c}\text { Temperatura } \\
\left({ }^{\circ} \mathrm{C}\right)\end{array}$ & $\mathbf{p H}$ & $\begin{array}{c}\text { Conductividad } \\
(\mu \mathrm{S} / \mathrm{cm})\end{array}$ \\
\hline Balsa Blanca & Almería & 26,0 & 8,6 & 166 \\
\hline Balsa de Barjalí & Almería & 22,0 & 8,8 & 198 \\
\hline Balsa de Caparidal & Almería & 24,0 & 8,3 & 177 \\
\hline Balsa de Punta Entinas & Almería & & & \\
\hline Balsa del Sabinar & Almería & 19,2 & 9,0 & 102 \\
\hline Embalse de Alcalá del Río & Sevilla & 25,0 & 7,8 & 880 \\
\hline Embalse de Béznar & Granada & 16,5 & 8,3 & 531 \\
\hline Embalse de Cala & Sevilla & 28,2 & 9,4 & 235 \\
\hline Embalse de Calderón & Sevilla & 22,0 & 7,8 & 5300 \\
\hline Embalse de Canales & Granada & 17,0 & 8,2 & 166 \\
\hline Embalse de Cubillas & Granada & & & \\
\hline Embalse de Guadalén & Jaén & 26,0 & 8,8 & 440 \\
\hline Embalse de Guadalhorce & Málaga & 13,0 & 8,1 & 11890 \\
\hline Embalse de Guadalmellato & Córdoba & 26,6 & 8,7 & 260 \\
\hline Embalse de Iznájar & Córdoba & 19,9 & 8,1 & 1025 \\
\hline Embalse de La Marciaga & Sevilla & & & \\
\hline Embalse de los Bermejales & Granada & 22,4 & 8,4 & 540 \\
\hline Embalse de los Hurones & Cádiz & 18,7 & 8,1 & 537 \\
\hline Embalse de Mengíbar & Jaén & & & \\
\hline Embalse de Panzacola & Jaén & 27,1 & 8,3 & 270 \\
\hline Embalse de Puente Nuevo & Córdoba & 27,0 & 8,9 & 370 \\
\hline Embalse de Rúmblar & Jaén & 24,6 & 7,9 & 270 \\
\hline Embalse de Vadomojón & Jaén & 20,0 & 8,1 & 2160 \\
\hline Embalse del Almanzora & Almería & 13,7 & 8,1 & 2205 \\
\hline Laguna Amarga & Córdoba & 25,2 & 8,4 & 7920 \\
\hline Laguna de Aguas Verdes & Granada & & & \\
\hline Laguna de Consuegra & Sevilla & 28,1 & 8,3 & 14350 \\
\hline Laguna de Garcíez & Jaén & 18,8 & 8,9 & 3100 \\
\hline Laguna de Garrucha & Almería & 21,0 & 7,7 & 7600 \\
\hline Laguna de las Pedrizas & Málaga & 26,1 & 8,0 & 2720 \\
\hline Laguna de Medina & Cádiz & 30,3 & 7,0 & 6750 \\
\hline Laguna de Mojácar & Almería & 22,0 & 7,7 & 8100 \\
\hline
\end{tabular}

Tabla 1 


\begin{tabular}{llccc}
\hline \multicolumn{1}{c}{ Localidad } & \multicolumn{1}{c}{ Provincia } & $\begin{array}{c}\text { Temperatura } \\
\left({ }^{\mathbf{}} \mathbf{C}\right)\end{array}$ & $\mathbf{p H}$ & $\begin{array}{c}\text { Conductividad } \\
(\boldsymbol{\mu S} / \mathbf{c m})\end{array}$ \\
\hline Laguna de Montellano & Cádiz & 30,3 & 7,0 & 12380 \\
Laguna de Río Seco Inferior & Granada & & & \\
Laguna de Santa Olalla & Huelva & 30,3 & 8,7 & 6480 \\
Laguna de Zóñar & Córdoba & 23,2 & 8,5 & 2650 \\
Laguna del Pajarejo & Jaén & 19,3 & 7,9 & 2840 \\
Laguna del Sopetón & Huelva & 29,0 & 8,5 & 13800 \\
Laguna Dulce & Huelva & 23,3 & 8,0 & 4230 \\
Laguna El Portil & Huelva & 30,8 & 8,7 & 1550 \\
Laguna Escalera & Sevilla & 18,2 & 8,3 & 1150 \\
Laguna Grande & Jaén & 18,1 & 8,3 & 791 \\
Laguna Grande de Archidona & Málaga & 27,4 & 8,0 & 3830 \\
\hline
\end{tabular}

Tabla 1 (continuación). Datos físico-químicos en las localidades muestreadas. Physico-chemical data in sampled localities.

lugares de especial interés (Parques Nacionales, Reservas Naturales,...). Las lagunas estudiadas son sistemas de agua someros en los que en principio no se establece termoclina, algunas de ellas llegando incluso a desecarse durante parte del año. Su régimen hídrico es muy variable dependiendo del volumen de precipitaciones anuales. En cuanto a los sistemas artificiales, donde en verano suelen desarrollarse las algas verdes cocales, hemos procurado muestrear embalses representativos de distintos grados tróficos y de mineralización. En este catálogo incluimos las especies citadas por otros autores hasta la fecha en Andalucía, junto a las halladas en nuestros muestreos. Algunas de ellas se han encontrado por primera vez en la comunidad andaluza o incluso en España o el conjunto de la Península Ibérica.

\section{METODOLOGÍA}

Para llevar a cabo este estudio nos hemos basado en recolecciones efectuadas durante los años 2004 a 2006, en las que se ha pretendido muestrear en el mayor número posible de sistemas acuáticos continentales de Andalucía (tab. 1), y en las citas bibliográficas existentes. Las muestras se obtuvieron tanto en las zonas litorales como en las de mayor profundidad. En ambos ambientes se tomaron dos muestras diferentes: una de carácter cuantitativo, en la que se confinó un volumen determinado de agua en un recipiente de $100 \mathrm{ml}$, fijándola posteriormente con lugol acético, destinada básicamente al estudio de estructura y dinámica de las poblaciones (Fanés Treviño \& Sánchez Castillo, en preparación); y otra tomada con una red de plancton de $40 \mu \mathrm{m}$ de tamaño de poro, que se fijó con formaldehído al $4 \%$. Las muestras se estudiaron en un microscopio invertido Zeiss Axiovert 35 dotado de objetivo de 100x utilizando el contraste de fase y las tinciones específicas necesarias cuando fue preciso.

Para la determinación de los taxa recolectados durante nuestro trabajo de campo han sido utilizadas la obra de Komárek y Fott (1983) así como las distintas monografías existentes de cada uno de los grupos tratados 
(Philipose, 1967; Komárková-Legnerová, 1969; Reháková, 1969; Hindák, 1977, 1980, 1984, 1988, 1990; Komárek \& Perman, 1978; Comas, 1996; Komárek \& Marvan, 1992; Komárek \& Jankovská, 2001; entre otros). Cada una de estas muestras fue fijada y depositada en el Herbario de la Universidad de Granada (GDAC).

El catálogo florístico viene ordenado, en sentido general, según la clasificación utilizada por Komárek y Fott (1983), puesto que incluye todas las algas verdes cocales y la clasificación actualizada se encuentra aún en sus inicios, aunque hemos aplicado los cambios basados en criterios más recientes que ya han sido aceptados. De cada taxon se muestran las localidades, diferenciando la provincia, y las referencias bibliográficas existentes. Finalmente se resaltan algunas especies por tratarse de nuevas citas.

\section{RESULTADOS \\ CATÁLOGO FLORÍSTICO}

\section{PALMELLACEAE}

Planktosphaeria gelatinosa G. M. Smith 1918

ALMERÍA: Balsa del Sabinar. GRANADA: Laguna de Zafarraya (de la Rosa, 1992).

Sphaerocystis planctonica (Koršikov) Bourrelly 1966

SEVILLA: Embalse de la Minilla (Casco, 1990).

\section{Sphaerocystis schroeteri Chodat 1897}

CÁDIZ: Embalse de Guadalcacín, Embalse de La Breña (Margalef et al., 1976). CÓRDOBA: Embalse de Guadalmellato, Embalse El Retortillo, Embalse de Bembézar, Embalse de Iznájar (Margalef et al., 1976). HUELVA: Embalse de Aracena (Margalef et al., 1976). JAÉN: Embalse de Guadalén, Embalse de Rúmblar, Embalse de Jándula, Embalse de Tranco de Beas, Embalse de Guadalmena (Margalef et al., 1976). MÁLAGA: Embalse Conde de Guadalhorce (Margalef et al., 1976). SEVILLA: Embalse del Pintado, Embalse de La Minilla,
Embalse de Cala (Margalef et al., 1976); Embalse de La Minilla (Toja, 1980; Toja et al., 1983).

Muchas poblaciones planctónicas, ampliamente distribuidas, que se reproducen exclusivamente por autósporas han sido identificadas por error como Sphaerocystis schroeteri, que se reproduce mayormente por zoósporas. Se trataba en realidad de Coenococcus fottii.

\section{CHARACIACEAE}

Ankyra ancora (G. M. Smith) Fott 1957

CÓRDOBA: Embalse El Retortillo (Margalef et al., 1976).

Ankyra judayi (G. M. Smith) Fott 1957

ALMERÍA: Embalse del Almanzora; Balsa de Barjalí. CÁDIZ: Laguna de Medina; Embalse de los Hurones. CÓRDOBA: Embalse de Guadalmellato; Embalse de Iznájar. GRANADA: (Cambra et al., 1989); Embalse de Canales. JAÉN: Laguna Grande; Embalse de Panzacola. SEVILLA: Embalse de Alcalá del Río; Laguna de Consuegra.

Ankyra lanceolata (Koršikov) Fott 1957

= Lanceola spatulifera (Koršikov) Hindák 1988

ALMERÍA: Embalse del Almanzora. CÁDIZ: Embalse de los Hurones. CÓRDOBA: Embalse de Guadalmellato. JAÉN: Embalse de Panzacola.

\section{Bicuspidella sessilis Fott 1953}

SEVILLA: Embalse de La Minilla (Casco et Toja, 1994).

Characium acuminatum Braun in Kützing 1849

SEVILLA: Embalse de La Minilla (Casco et Toja, 1994).

Korshikoviella gracilipes (Lambert) Silva 1959

GRANADA: Río Seco Superior, Río Seco Inferior (Sierra Nevada) (Sánchez Castillo et al., 1989); Río Seco (Sierra Nevada) (Sánchez Castillo, 1987b; Pérez Martínez et al., 2001; Barea Arco et al., 2001; Pérez Martínez et al., 2007); Río Seco, Río Seco Inferior, Río Seco Superior y Veleta (Sierra Nevada) (Sánchez Castillo, 1986); Lagunas de Sierra Nevada (Sánchez Castillo, 1988).

Korshikoviella limnetica (Lemmermann) Silva 1959 
JAÉN: (Avilés et González Ramos, 1981).

Paradoxia multiseta Svirenko 1928

CÓRDOBA: Embalse de Guadalmellato.

Pseudoschroederia antillarum (Komárek) Hegewald et Schnepf 1986

= Schroederia antillarum Komárek 1983

CÁDIZ: Embalse de los Hurones. JAÉN:

Embalse de Guadalén.

Pseudoschroederia robusta (Koršikov) Hegewald et Schnepf 1986

= Schroederia robusta Koršikov 1983

CÁDIZ: Laguna de Medina; Embalse de los Hurones. CÓRDOBA: Embalse de Puente Nuevo. JAÉN: Embalse de Guadalén. SEVILLA: Laguna Escalera.

Schroederia setigera (Schröder) Lemmermann 1898

CÁDIZ: "La Cortadura", Río San Pedro (González Guerrero, 1946).

\section{TREUBARIACEAE}

Treubaria schmidlei (Schröder) Fott et Kovácik 1975

CÁDIZ: Embalse de los Hurones. JAÉN: Embalse de Guadalén. SEVILLA: Embalse de Alcalá del Río.

Treubaria triappendiculata Bernard 1908

HUELVA: Embalse de Aracena (Toja, 1976).

SEVILLA: Embalse de La Minilla (Toja, 1976).

\section{GOLENKINIACEAE}

\section{Golenkinia radiata Chodat 1894}

CÁDIZ: (Avilés et González Ramos, 1981);

Embalse de los Hurones. GRANADA: Alhambra (Sánchez Castillo, 1983). HUELVA: Laguna Dulce (Doñana) (Margalef, 1976).

\section{HYDRODICTYACEAE}

Hydrodictyon reticulatum (Linnaeus) Lagerheim 1883

CÁDIZ: (Colmeiro, 1867; Bellón, 1942).

SEVILLA: (González Fragoso, 1893; Lázaro,
1896).

Pediastrum biradiatum Meyen 1829

HUELVA: (Avilés et González Ramos, 1981).

Pediastrum boryanum var. boryanum (Turpin) Meneghini 1840

CÁDIZ: (Avilés et González Ramos, 1981). CÓRDOBA: (Avilés et González Ramos, 1981); Embalse de Celemín, Embalse de Guadalcacín (Margalef et al., 1976); Laguna de Zóñar. GRANADA: Facultad de Filosofía y Letras, Parque del Triunfo, Cartuja (Sánchez Castillo, 1983); Laguna de Yeguas, Laguna Virgen Superior, Laguna Virgen Media, Laguna de Aguas Verdes, Laguna Gemela, Laguna de Majano, Laguna de San Juan (Sánchez Castillo, 1988); Laguna Virgen Superior, Laguna Virgen Media, Laguna de Aguas Verdes, Laguna de Río Seco, Laguna Gemela, Laguna de Majano (Sánchez Castillo et al., 1989); (Cambra et al., 1989); Embalse de Moraleda (de la Rosa, 1992); Embalse de los Bermejales (Pérez Martínez, 1992); Embalse de Béznar; Laguna de Río Seco Inferior; Laguna de Aguas Verdes; Embalse de Cubillas. HUELVA: Laguna Dulce, Lagunita en las Dunas, Laguna Grande, Laguna de Santa Olalla, Charco del Toro (Margalef, 1976); Embalse de Aracena (Margalef et al., 1976); (Avilés et González Ramos, 1981); Laguna de Santa Olalla. JAÉN: (Avilés et González Ramos, 1981); Embalse de Jándula (Margalef et al., 1976); Embalse de Vadomojón; Laguna Grande. MÁLAGA: (Avilés et González Ramos, 1981); Laguna Grande de Archidona. SEVILLA: Embalse de Torre del Águila, Embalse de La Minilla, Embalse de Cala, Embalse del Pintado (Margalef et al., 1976); (Avilés et González Ramos, 1981); Embalse de La Minilla (Casco et Toja, 1994); Laguna Escalera.

Pediastrum boryanum var. brevicorne A. Braun 1855

GRANADA: Embalse de Cubillas. HUELVA: Laguna en Punta Umbría (Doñana) (Margalef, 1976). MÁLAGA: (Margalef, 1944).

Pediastrum boryanum var. cornutum (Raciborski) Sulek 1969

GRANADA: Laguna de Yeguas, Laguna Virgen Superior, Laguna Virgen Media, Laguna de Aguas Verdes, Laguna de Río Seco, Laguna Gemela, 
Laguna Cuarta (Sánchez Castillo, 1988).

Pediastrum boryanum var. longicorne Reinsch 1867

CÁDIZ: Embalse de Bornos (Margalef et al., 1976). GRANADA: Embalse de los Bermejales (Sánchez Castillo et Gil, 1982); Parque del Triunfo (Sánchez Castillo, 1983). MÁLAGA: Embalse de Guadalhorce, Embalse de Guadalteba (Margalef et al., 1976).

Pediastrum braunii Wartmann 1862

GRANADA: Laguna de Aguas Verdes, Gemela, Virgen Media, Virgen Superior (Sierra Nevada) (Sánchez Castillo, 1986); Lagunas de Sierra Nevada (Sánchez Castillo, 1988).

\section{Pediastrum duplex Meyen 1829}

CÁDIZ: (González Guerrero, 1928); Embalse de Bornos, Embalse de Celemín, Embalse de Guadalcacín (Margalef et al., 1976); (Avilés et González Ramos, 1981); Embalse de los Hurones. CÓRDOBA: Embalse El Retortillo, Embalse de Guadalmellato, Embalse de Iznájar (Margalef et al., 1976); (Avilés et González Ramos, 1981); Embalse de Iznájar. GRANADA: Embalse de Béznar. HUELVA: Embalse de Aracena (Margalef et al., 1976); (Avilés et González Ramos, 1981); Laguna de Santa Olalla; Laguna Dulce; Laguna El Portil. JAÉN: Embalse de Tranco de Beas, Embalse de Guadalén, Embalse de Rúmblar, Embalse de Jándula (Margalef et al., 1976); (Cambra et al., 1989); Embalse de Vadomojón; Laguna Grande; Embalse de Guadalén. MÁLAGA: Embalse de Guadalteba (Margalef et al., 1976). SEVILLA: Embalse de Torre del Águila (Margalef et al., 1976); (Avilés et González Ramos, 1981); Embalse de La Minilla (Toja, 1980; Casco et Toja, 1994); Embalse de Alcalá del Río.

Pediastrum duplex var. gracillimum W. et G. S. West 1895

CÓRDOBA: Embalse El Retortillo (Margalef et al., 1976). HUELVA: Laguna de Santa Olalla, Charco del Toro (Doñana) (Margalef, 1976).

Pediastrum simplex var. simplex Meyen 1829

CÁDIZ: Embalse de Celemín, Embalse de Bornos, Embalse de Guadalcacín, Embalse de Guadarranque (Margalef et al., 1976); (Avilés et González Ramos, 1981); Embalse de los Hurones.
CÓRDOBA: Embalse de Guadanuño, Embalse de La Breña, Embalse de Bembézar, Embalse El Retortillo, Embalse de Guadalmellato (Margalef et al., 1976); (Avilés et González Ramos, 1981); Embalse de Iznájar; Embalse de Puente Nuevo; Embalse de Guadalmellato. GRANADA: Embalse de los Bermejales (Margalef et al., 1976); Embalse de Moraleda (de la Rosa, 1992). HUELVA: Lagunas del Parque Nacional de Doñana (Margalef, 1976); Embalse de Aracena (Margalef et al., 1976); Laguna Dulce (Doñana) (Margalef, 1976); (Avilés et González Ramos, 1981); Laguna El Portil. JAÉN: Embalse de Guadalén, Embalse de Guadalmena (Margalef et al., 1976). (Avilés et González Ramos, 1981); Embalse de Rúmblar; Embalse de Panzacola; Laguna Grande. MÁLAGA: Laguna Grande de Archidona. SEVILLA: Embalse de Cala, Embalse de La Minilla, Embalse del Pintado, Embalse de Torre del Águila (Margalef et al., 1976); Embalse de La Minilla (Toja, 1980); (Avilés et González Ramos, 1981); Laguna Escalera; Embalse de Alcalá del Río.

Pediastrum simplex var. biwaense Fukushima 1956

CÓRDOBA: Embalse de Guadalmellato. JAÉN: Embalse de Guadalén; Embalse de Rúmblar; Embalse de Panzacola; Embalse de Mengíbar. SEVILLA: Embalse de Cala.

Pediastrum simplex var. echinulatum Wittrock 1883

JAÉN: Embalse de Guadalén.

Pediastrum simplex var. sturmii (Reinsch) Wolle 1887

GRANADA: (Cambra et al., 1989). SEVILLA: Embalse de La Minilla (Casco et Toja, 1994).

Pediastrum tetras (Ehrenberg) Ralfs 1844

ALMERÍA: Balsa de Punta Entinas. CÁDIZ: (Avilés et González Ramos, 1981). CÓRDOBA: (Avilés et González Ramos, 1981). GRANADA: Laguna de Virgen Media (Sierra Nevada) (Sánchez Castillo, 1986); Llanura marginal que rodea a la $7^{\text {a }}$ Laguna (Mulhacén, Sierra Nevada) (González Guerrero, 1981); Lagunas de Sierra Nevada (Sánchez Castillo, 1988); Embalse de Moraleda (de la Rosa, 1992). HUELVA: Laguna de Santa Olalla, El Rocío (Doñana) (Margalef, 1976); Embalse de Aracena 
(Margalef et al., 1976); (Avilés et González Ramos, 1981); Laguna de Sopetón. JAÉN: (González Guerrero, 1928).

\section{MICRACTINIACEAE}

Dicellula geminata (Printz) Koršikov 1953

JAÉN: Embalse de Guadalén.

Golenkiniopsis parvula (Voronichin) Koršikov 1953

GRANADA: Laguna de Alcantar (de la Rosa, 1992).

Micractinium crassisetum Hortobágyi 1973

SEVILLA: Embalse de Alcalá del Río.

\section{Micractinium pusillum Fresenius 1858}

CÁDIZ: Embalse de los Hurones. GRANADA: Alhambra (Sánchez Castillo, 1983); Embalse de Moraleda (de la Rosa, 1992); Embalse de los Bermejales (Pérez Martínez, 1992). JAÉN: Embalse de Guadalén.

\section{BOTRYOCOCCACEAE}

\section{Botrycoccus braunii Kützing 1849}

ALMERÍA: Balsa del Sabinar. CÁDIZ: Embalse de Celemín (Margalef et al., 1976). CÓRDOBA: Embalse El Retortillo (Margalef et al., 1976). HUELVA: Marisma de Hinojos, Laguna de Punta Umbría (Doñana) (Margalef, 1976). JAÉN: Embalse de Guadalén (Margalef et al., 1976); Laguna de Garcíez. MÁLAGA: Embalse de Guadalteba, Embalse Conde de Guadalhorce (Margalef et al., 1976); Laguna Chica de Archidona (de la Rosa, 1992).

Botryococcus terribilis Komárek et Marvan 1992 ALMERÍA: Balsa de Barjalí. CÁDIZ: Laguna de Montellano; Embalse de los Hurones. CÓRDOBA: Laguna de Zóñar; Laguna Amarga; Embalse de Iznájar. MÁLAGA: Laguna Grande de Archidona.

Dichotomococcus curvatus Koršikov 1939

JAÉN: Embalse de Guadalén. SEVILLA: Embalse de Alcalá del Río.

Dictyosphaerium chlorelloides (Nauman) Komárek et Perman 1978
GRANADA: Laguna de Río Seco (Pérez Martínez et al., 2001; Barea Arco et al., 2001; Pérez Martínez et al., 2007); Laguna de La Caldera (Delgado Molina et al., 2009).

Dictyosphaerium ehrenbergianum Nägeli 1849

JAÉN: Embalse de Guadalén. HUELVA: Charca del Toro (Doñana) (Margalef, 1976).

Dictyosphaerium elongatum Hindák 1977 CÓRDOBA: Embalse de Puente Nuevo.

Dictyosphaerium pulchellum Wood 1872

ALMERÍA: Laguna Honda (Albuferas de Adra) (Sánchez Castillo, 1987a). CÁDIZ: Embalse de Celemín, Embalse de Bornos, Embalse de Guadalcacín (Margalef et al., 1976). CÓRDOBA: Embalse de Guadalmellato, Embalse El Retortillo (Margalef et al., 1976). GRANADA: Alhambra (Sánchez Castillo, 1983); Laguna del Padul (Pérez Martínez et Sánchez Castillo, 1990); Embalse de Moraleda, Laguna de Alcantar (de la Rosa, 1992). HUELVA: Embalse de Aracena (Margalef et al., 1976). JAÉN: Embalse de Guadalén, Embalse de Rúmblar (Margalef et al., 1976). MÁLAGA: Embalse de Guadalteba (Margalef et al., 1976). SEVILLA: Embalse del Pintado, Embalse de Torre del Águila, Embalse de La Minilla (Margalef et al., 1976).

Dictyosphaerium pulchellum var. minutum Deflandre 1926

MÁLAGA: Laguna de las Pedrizas.

Dictyosphaerium subsolitarium Van Goor 1924

ALMERÍA: Embalse del Almanzora. GRANADA: Embalse de los Bermejales (Pérez Martínez, 1992; Pérez Martínez et Cruz Pizarro, 1995). SEVILLA: Embalse de Cala.

Dictyosphaerium tetrachotomum Printz 1914

ALMERÍA: Balsa de Caparidal; Embalse del Almanzora. CÁDIZ: Embalse de los Hurones. GRANADA: Embalse de Canales. SEVILLA: Embalse de La Marciaga.

Lobocystis cf. planctonica Fott $1975 ?$

CÓRDOBA: Embalse de Puente Nuevo.

La población estudiada presenta células de morfología y dimensiones algo distintas de la 
típica $L$. planctonica, por lo que no confirmamos su identificación.

Quadricoccus ellipticus Hortobágyi 1973

CÓRDOBA: Embalse de Guadalmellato. SEVILLA: Embalse de Alcalá del Río.

\section{RADIOCOCCACEAE}

Coenococcus fottii Hindák 1977

= Eutetramorus fottii (Hindák) Komárek $1979=$ Coenochloris polycocca $($ Koršikov) Hindák 1984

CÁDIZ: Embalse de los Hurones. CÓRDOBA: Embalse de Guadalmellato; Embalse de Iznájar. GRANADA: Laguna Gemela (Sierra Nevada) (Sánchez Castillo, 1986); Lagunas de Sierra Nevada (Sánchez Castillo, 1988); Embalse de Canales. JAÉN: Embalse de Panzacola.

Coenocystis tapasteana Komárek 1983

GRANADA: Embalse de los Bermejales.

Gloeocystis polydermatica (Kützing) Hindák 1978 SEVILLA: (Delás, 1883).

\section{Pectodictyon cubicum Taft 1945}

GRANADA: Facultad de Filosofía y Letras (Sánchez Castillo, 1983).

Radiococcus nimbatus (De Wildemann) Schmidle 1902

MÁLAGA: Embalse de Guadalhorce (Armengol et al., 1990).

\section{OOCYSTACEAE}

Eremosphaera eremosphaeria (G. M. Smith) R. M. Smith et Bold 1966

SEVILLA: Embalse de La Minilla (Casco et Toja, 1994).

Eremosphaera gigas (Archer) Fott et Kalina 1962 = Oocystis gigas Archer 1877

GRANADA: Charcos turberales inmediatos a la $7^{\text {a }}$ Laguna (Mulhacén, Sierra Nevada) (González Guerrero, 1981).

Franceia ovalis (Francé) Lemmermann 1898 CÁDIZ: Embalse de los Hurones.
Gloeotaenium loitlesbergerianum Hansgirg 1890 ALMERÍA: Balsa de Punta Entinas.

Lagerheimia balatonica (Scherfell) Hindák 1978

$=$ Chodatella balatonica Scherfell in Kol 1938

HUELVA: Laguna de Santa Olalla (Doñana) (Margalef, 1976).

Lagerheimia ciliata (Lagerheim) Chodat 1895

$=$ Chodatella ciliata (Lagerheim) Lemmermann 1898

CÁDIZ: Embalse de Bornos (Margalef et al., 1976); (Avilés et González Ramos, 1981); Embalse de los Hurones. GRANADA: Embalse de Canales. HUELVA: Embalse de Aracena (Toja et al., 1983). SEVILLA: Embalse de La Minilla (Margalef et al., 1976); Embalse de La Minilla (Casco et Toja, 1994).

Lagerheimia genevensis (Chodat) Chodat 1895

= Chodatella genevensis (Chodat) Ley 1948

HUELVA: (Avilés et González Ramos, 1981). SEVILLA: Embalse de La Minilla (Casco et Toja, 1994).

Lagerheimia longiseta (Lemmermann) Printz 1913

= Chodatella longiseta Lemmermann 1898

SEVILLA: Embalse de La Minilla (Casco et Toja, 1994).

Lagerheimia quadriseta (Lemmermann) G. M. Smith 1926

= Chodatella quadriseta Lemmermann 1898

CÁDIZ: Embalse de Bornos (Margalef et al., 1976); Embalse de los Hurones. CÓRDOBA: Embalse de Iznájar. GRANADA: Embalse de Cubillas. HUELVA: Embalse de Aracena (Toja et al., 1983). JAÉN: Embalse de Mengíbar. MÁLAGA: Embalse de Guadalhorce. SEVILLA: Embalse de La Minilla (Toja, 1980).

Lagerheimia subsalsa Lemmermann 1898

= Chodatella subsalsa Lemmermann 1898

CÁDIZ: Embalse de los Hurones. CÓRDOBA: (Avilés et González Ramos, 1981). GRANADA: Embalse de Cubillas. HUELVA: Laguna de Santa Olalla (Doñana) (Margalef, 1976). JAÉN: Embalse de Guadalén. 
Lagerheimia wratislaviensis Schröder 1897 1948

$=$ Chodatella wratislaviensis (Schröder) Ley

HUELVA: Laguna Dulce (Doñana) (Margalef, 1976).

Nephrocytium agardhianum Nägeli 1849

GRANADA: Embalse de Canales. HUELVA:

El Ojillo, Marisma de Hinojos (Doñana) (Margalef, 1976).

Oocystis elliptica W. West 1892

GRANADA: Charcos del Mulhacén (Sierra

Nevada) (González Guerrero, 1981).

Oocystis lacustris Chodat 1897

= Oocystella lacustris (Chodat) Hindák 1988

ALMERÍA: Laguna Honda (Albufera de Adra) (Carrillo et al., 1987). CÁDIZ: Embalse de Bornos (Margalef et al., 1976); Embalse de los Hurones. CÓRDOBA: Embalse de La Breña, Embalse El Retortillo (Margalef et al., 1976). GRANADA: Lagunas de Sierra Nevada (Morales Baquero et al., 1992); Río Seco superior, Río Seco, Gemela, Majano (Sierra Nevada) (Sánchez Castillo et al., 1989); Laguna de La Caldera, Gemela, Río Seco, Río Seco superior y Virgen Media (Sierra Nevada) (Sánchez Castillo, 1986); Lagunas de Sierra Nevada (Sánchez Castillo, 1988); Laguna de La Caldera (Carrillo et al., 1990); Embalse de los Bermejales (Pérez Martínez, 1992; Pérez Martínez et Cruz Pizarro, 1995); Embalse de Béznar. HUELVA: Embalse de Aracena (Margalef et al., 1976). JAÉN: Embalse de Guadalmena, Embalse de Guadalén, Embalse de Tranco de Beas, Embalse de Rúmblar (Margalef et al., 1976). MÁLAGA: Embalse de Guadalhorce, Embalse de La Concepción, Embalse Conde de Guadalhorce (Margalef et al., 1976); Embalse de Guadalteba, Embalse de Guadalhorce (Armengol et al., 1990). SEVILLA: Embalse de La Minilla (Margalef et al., 1976; Toja, 1980; Toja et al., 1983).

Oocystis marssoni Lemmermann 1898

$=$ Oocystella marssonii (Lemmermann) Hindák 1988

CÓRDOBA: Laguna de Zóñar; Embalse de Guadalmellato; Embalse de Iznájar. GRANADA: Laguna de San Juan (Sierra Nevada) (Sánchez Castillo, 1986); Embalse de los Bermejales (Pérez
Martínez, 1992); Lagunas de Sierra Nevada (Sánchez Castillo, 1988); Embalse de los Bermejales (Pérez Martínez et Cruz Pizarro, 1995); Embalse de Béznar; Embalse de Canales. HUELVA: (Toja et Casco, 1991). JAÉN: Laguna Grande; Embalse de Guadalén; Embalse de Rúmblar; Embalse de Vadomojón; Embalse de Mengíbar. SEVILLA: Embalse de La Minilla (Casco et Toja, 1994); Embalse de La Marciaga; Laguna Escalera.

Oocystis parva W. et G. S. West 1898

= Oocystella parva (W. et G. S. West) Hindák $1988=$ Oocystis lacustris Chodat sensu Hindák 1980

CÁDIZ: Laguna de Medina; Embalse de los Hurones. CÓRDOBA: Embalse de Iznájar. GRANADA: Laguna de Charco Negro, Laguna de Zafarraya, Embalse de Moraleda (de la Rosa, 1992); Embalse de los Bermejales. JAÉN: (Aboal, 1996); Embalse de Panzacola; Embalse de Mengíbar. MÁLAGA: Laguna Grande de Archidona, Laguna Chica de Archidona (de la Rosa, 1992). SEVILLA: Laguna de Calderón.

Oocystis pusilla Hansgirg 1890

GRANADA: Cartuja (Sánchez Castillo, 1983).

Oocystis solitaria Wittrock 1879

= Oocystella solitaria (Wittrock) Hindák 1988

ALMERÍA: Balsa de Caparidal. GRANADA: Embalse de los Bermejales (Sánchez Castillo et Gil, 1982); Facultad de Filosofía y Letras, Facultad de Ciencias (Sánchez Castillo, 1983); Laguna Virgen Media, Majano (Sierra Nevada) (Sánchez Castillo, 1986); Lagunas de Sierra Nevada (Sánchez Castillo, 1988). HUELVA: Laguna Grande (Dulce), Marisma del Guadalquivir, Laguna de Santa Olalla, Laguna de Punta Umbría (Doñana) (Margalef, 1976). JAÉN: Laguna de Garcíez. MÁLAGA: (Margalef, 1944); Laguna Grande de Archidona (de la Rosa, 1992).

Oocystis cf. solitaria Wittrock 1879

CÓRDOBA: Embalse de Iznájar.

A diferencia de la típica $O$. solitaria, las células de esta población no presentan siempre los típicos engrosamientos polares y suelen estar en colonias de 2 a 4 células, nunca aisladas, por lo que no podemos confirmar que se trate de esta especie. 
Oocystis submarina Lagerheim 1886

= Oocystella submarina (Lagerheim) Hindák 1988

GRANADA: Embalse de Canales. SEVILLA: Laguna Escalera.

Tetrachlorella alternans (G. M. Smith) Koršikov 1939

GRANADA: Embalse de Cubillas. JAÉN: Embalse de Rúmblar. MÁLAGA: Embalse de Guadalhorce.

Tetrachlorella incerta Hindák 1977

GRANADA: Embalse de los Bermejales (Pérez Martínez, 1992).

Tetrachlorella ornata Koršikov 1953

CÁDIZ: Embalse de los Hurones. JAÉN: Laguna Grande. MÁLAGA: Laguna Grande de Archidona.

\section{CHLORELLACEAE}

Ankistrodesmus bibraianus (Reinsch) Koršikov 1953

= Selenastrum bibraianum Reinsch 1867

GRANADA: Cabeza lagunar del nacimiento del Río Guadalfeo (Mulhacén, Sierra Nevada) (González Guerrero, 1981). HUELVA: Río Tinto (González Guerrero, 1950).

Ankistrodesmus falcatus (Corda) Ralfs 1848

CÁDIZ: Algeciras (Budde, 1929); Embalse de Guadarranque (Margalef et al., 1976); (Avilés et González Ramos, 1981). CÓRDOBA: Embalse El Retortillo (Margalef et al., 1976); (Avilés et González Ramos, 1981). GRANADA: Embalse de los Bermejales (Margalef et al., 1976); Charcos turberales del margen de la $7^{\mathrm{a}}$ Laguna (Mulhacén, Sierra Nevada) (González Guerrero, 1981); Laguna Gemela, Majano (Sierra Nevada) (Sánchez Castillo et al., 1989); Laguna de Aguas Verdes, Gemela, Majano, Virgen Media (Sierra Nevada) (Sánchez Castillo, 1986, 1988). HUELVA: Río Tinto (González Guerrero, 1950); Laguna Dulce, Laguna Grande (Dulce), Acebuche de Matalascañas, Marisma del Guadalquivir, Laguna de Santa Olalla, Laguna del Taraje, Charca del Toro, El Rocío, El Rocío tierras inundadas (Doñana) (Margalef, 1976); (Avilés et González Ramos, 1981). JAÉN: (González
Guerrero, 1928); Embalse de Guadalmena (Margalef et al., 1976); (Avilés et González Ramos, 1981). MÁLAGA: Embalse de Guadalteba (Margalef et al., 1976). SEVILLA: Embalse de Torre del Águila, Embalse del Pintado (Margalef et al., 1976); (Avilés et González Ramos, 1981).

\section{Ankistrodesmus nannoselene Skuja 1948}

SEVILLA: Embalse de La Minilla (Toja, 1980).

Ankistrodesmus stipitatus (Chodat) KomárkováLegnerová 1969

SEVILLA: Embalse de Alcalá del Río.

Chlorella minutissima Fott et Nováková 1969 CÓRDOBA: Embalse de Iznájar.

\section{Chlorella vulgaris Beijerinck 1890}

GRANADA: San Jerónimo (Sánchez Castillo, 1983); Embalse de los Bermejales (Pérez Martínez, 1992); Embalse de los Bermejales (Pérez Martínez et Cruz Pizarro, 1995). SEVILLA: Embalse de La Minilla (Toja, 1980; Toja et al., 1983).

Chlorolobion saxatile (Komárková-Legnerová) Komárek 1979 JAÉN: Río Madera (Aboal, 1988).

Closteriopsis acicularis (G. M. Smith) Belcher et Swale 1962

HUELVA: Laguna de Sopetón.

Esta especie había sido citada en la Laguna del Padul (Granada) por Pérez Martínez y Sánchez Castillo (1990), pero hubo un error en su identificación, se trataba en realidad de una especie de Closteriopsis (Sánchez Castillo, com. pers.).

Kirchneriella contorta (Schmidle) Bohlin 1897

= Pseudokirchneriella contorta (Schmidle) Hindák $1990=$ Raphidocelis contorta (Schmidle) Marvan, Komárek et Comas 1984

CÁDIZ: Embalse de los Hurones.

Kirchneriella irregularis (G. M. Smith) Koršikov 1953

= Pseudokirchneriella irregularis (G. M.

Smith) Hindák 1990

SEVILLA: Embalse de Alcalá del Río.

Kirchneriella irregularis var. spiralis Koršikov 
1953

CÁDIZ: Embalse de los Hurones.

Kirchneriella lunaris (Kirchner) Möbius 1894

HUELVA: Embalse de Aracena (Margalef et al., 1976). JAÉN: Embalse de Rúmblar (Margalef et al., 1976). SEVILLA: Embalse de La Minilla (Toja et al., 1983).

Kirchneriella obesa (W. West) Schmidle 1893

CÓRDOBA: Embalse de Bembézar, Embalse

El Retortillo, Embalse de Guadalmellato (Margalef et al., 1976); (Avilés et González Ramos, 1981). GRANADA: Cartuja (Sánchez Castillo, 1983). JAÉN: Embalse de Rúmblar, Embalse de Jándula (Margalef et al., 1976); (Avilés et González Ramos, 1981). SEVILLA: Embalse de Torre del Águila (Margalef et al., 1976).

Kirchneriella rotunda (Koršikov) Hindák 1977

= Pseudokirchneriella rotunda (Koršikov)

Hindák $1990=$ Raphidocelis rotunda (Koršikov) Marvan, Komárek et Comas 1984

JAÉN: Embalse de Guadalén.

\section{Kirchneriella subcapitata Koršikov 1953}

= Pseudokirchneriella subcapitata (Koršikov)

Hindák $1990=$ Raphidocelis subcapitata (Schmidle)

Nygaard, Komárek, Kristiansen et Skulberg 1986

MÁLAGA: Laguna de las Pedrizas.

Monoraphidium arcuatum (Koršikov) Hindák 1970

JAÉN: Embalse de Guadalén. SEVILLA: Laguna Escalera; Embalse de La Marciaga.

\section{Monoraphidium caribeum Hindák 1970}

GRANADA: Laguna de Zafarraya (de la Rosa, 1992).

Monoraphidium circinale (Nygaard) Nygaard 1979

GRANADA: Laguna de Zafarraya, Laguna de Alcantar, Laguna de Charco Negro (de la Rosa, 1992).

Monoraphidium contortum (Thuret) KomárkováLegnerová 1969

ALMERÍA: Albufera de Adra (Carrillo et al., 1987; Sánchez Castillo, 1987a); Balsa de Barjalí;
Balsa Blanca; Embalse del Almanzora. CÁDIZ: (Avilés et González Ramos, 1981); Embalse de los Hurones. GRANADA: Laguna del Padul (Pérez Martínez et Sánchez Castillo, 1990); Laguna de Zafarraya (de la Rosa, 1992); Embalse de los Bermejales (Pérez Martínez, 1992); Embalse de Canales; Laguna de Río Seco Inferior. HUELVA: Laguna Dulce (Toja et al., 1991); Laguna de Sopetón; Laguna Dulce. JAÉN: Embalse de Vadomojón; Embalse de Guadalén; Embalse de Panzacola. MÁLAGA: Laguna de las Pedrizas; Laguna Grande de Archidona. SEVILLA: Laguna Escalera; Embalse de Alcalá del Río; Embalse de La Marciaga.

Monoraphidium convolutum (Corda) KomárkováLegnerová 1969

CÁDIZ: Laguna de Montellano. HUELVA: Laguna de Sopetón. MÁLAGA: (Margalef, 1944).

Monoraphidium griffithii (Berkeley) KomárkováLegnerová 1969

= Ankistrodesmus acicularis (Braun) Skuja

CÁDIZ: Embalse de Celemín (Margalef et al., 1976). SEVILLA: Embalse de La Minilla (Toja, 1980); Embalse El Gergal (Toja et al., 1983); Embalse de Alcalá del Río; Embalse de La Marciaga.

Monoraphidium irregulare (G. M. Smith) Komárková-Legnerová 1969

SEVILLA: (Avilés et González Ramos, 1981).

Monoraphidium komarkovae Nygaard 1979

ALMERÍA: Laguna Honda (Albufera de Adra) (Carrillo et al., 1987). GRANADA: Embalse de los Bermejales (Pérez Martínez, 1992); (Cambra et al., 1989). JAÉN: Embalse de Guadalén.

Monoraphidium minutum (Nägeli) KomárkováLegnerová 1969

= Selenastrum minutum (Nägeli) Collins 1907

ALMERÍA: Albufera de Adra (Carrillo et al., 1987). CÁDIZ: Laguna de Medina. GRANADA: Embalse de los Bermejales (Pérez Martínez, 1992); Laguna del Padul (Pérez Martínez et Sánchez Castillo, 1990). HUELVA: Laguna Grande, Charco del Toro, Laguna de Santa Olalla (Margalef, 1976). JAÉN: Embalse de Jándula (Margalef et al., 1976). SEVILLA: Laguna de Calderón. 
Monoraphidium obtusum (Koršikov) KomárkováLegnerová 1969

GRANADA: Albaicín (Sánchez Castillo, 1983).

Monoraphidium tortile (W. et G. S. West) Komárková-Legnerová 1969

GRANADA: Laguna de Zafarraya, Laguna de Alcantar (de la Rosa, 1992). SEVILLA: Embalse de La Minilla (Toja, 1980).

Scotiella tuberculata Bourrelly 1951

GRANADA: Laguna de la Caldera (Sierra Nevada) (Sánchez Castillo, 1986); Lagunas de Sierra Nevada (Sánchez Castillo, 1988).

Selenastrum capricornutum Printz 1914

HUELVA: Laguna de Santa Olalla (Doñana) (Margalef, 1976).

Tetraedron caudatum (Corda) Hansgirg 1888 CÓRDOBA: Embalse de Puente Nuevo. GRANADA: Laguna Gemela (Sierra Nevada) (Sánchez Castillo, 1986); Lagunas de Sierra Nevada (Sánchez Castillo, 1988); Embalse de Moraleda (de la Rosa, 1992). HUELVA: Lagunita en las Dunas, Charco del Toro (Margalef, 1976).

Tetraedron minimum (A. Braun) Hansgirg 1888 ALMERÍA: Albufera de Adra (Carrillo et al., 1987); Balsa de Punta Entinas. CÁDIZ: (Avilés et González Ramos, 1981); Embalse de los Hurones. CÓRDOBA: (Avilés et González Ramos, 1981); Embalse de Iznájar. GRANADA: Laguna La Caldera (Martínez Silvestre, 1977); Laguna de Alcantar (de la Rosa, 1992); Embalse de los Bermejales (Pérez Martínez, 1992); Embalse de Canales. HUELVA: Río Tinto (González Guerrero, 1950); Laguna de Santa Olalla (Doñana) (Margalef, 1976); (Avilés et González Ramos, 1981). JAÉN: (Avilés et González Ramos, 1981); Embalse de Panzacola; Embalse de Guadalén. MÁLAGA: (Avilés et González Ramos, 1981). SEVILLA: Embalse de La Minilla (Toja, 1980); Embalse El Gergal (Toja et al., 1983); (Avilés et González Ramos, 1981); Embalse de Alcalá del Río; Embalse de La Marciaga.

Tetraedron minimum var. scrobiculatum Lagerheim 1888

HUELVA: Laguna Dulce, Charca del Toro
(Doñana) (Margalef, 1976).

Tetraedron regulare Kützing 1845

CÁDIZ: Embalse de los Hurones. CÓRDOBA:

Embalse de Guadalmellato.

Tetraedron pentaedricum W. et G. S. West 1895

GRANADA: Laguna de Alcantar (de la Rosa, 1992).

Tetraedron trigonum (Nägeli) Hansgirg 1888

HUELVA: Charca del Toro (Doñana) (Margalef, 1976).

\section{SCENEDESMACEAE}

Actinastrum aciculare Playfair 1917

GRANADA: Laguna de Zafarraya, Embalse de Moraleda (de la Rosa, 1992).

Actinastrum hantzschii var. hantzschii Lagerheim 1882

ALMERÍA: Balsa de Caparidal. CÓRDOBA: Embalse de Puente Nuevo. GRANADA: Laguna Hondera (Sierra Nevada) (Sánchez Castillo, 1986). Lagunas de Sierra Nevada (Sánchez Castillo, 1988). HUELVA: Charco del Toro (Doñana) (Margalef, 1976). JAÉN: Embalse de Guadalén. MÁLAGA: Embalse de Guadalhorce (Margalef et al., 1976). SEVILLA: Embalse de Alcalá del Río.

Actinastrum hantzschii var. subtile Woloszynska 1911

ALMERÍA: Embalse del Almanzora. GRANADA: Laguna de Zafarraya, Embalse de Moraleda (de la Rosa, 1992).

Coelastrum astroideum De-Notaris 1867

JAÉN: Embalse de Vadomojón; Embalse de Guadalén. SEVILLA: Laguna Escalera.

\section{Coelastrum indicum Turner 1892}

CÁDIZ: Embalse de los Hurones. CÓRDOBA: Embalse de Puente Nuevo; Embalse de Iznájar. GRANADA: Laguna de Aguas Verdes.

Coelastrum microporum Nägeli in A. Braun 1855

CÁDIZ: Embalse de Celemín, Embalse de Bornos (Margalef et al., 1976); (Avilés et González Ramos, 1981). CÓRDOBA: Embalse de 
Guadalmellato, Embalse de La Breña (Margalef et al., 1976); (Avilés et González Ramos, 1981). GRANADA: Laguna Gemela, Laguna de San Juan (Sánchez Castillo, 1988); Embalse de Moraleda (de la Rosa, 1992). HUELVA: Laguna de Santa Olalla (Margalef, 1976); Embalse de Aracena (Margalef et al., 1976; Toja et al., 1983); (Avilés et González Ramos, 1981). JAÉN: Embalse de Tranco de Beas, Embalse de Rúmblar, Embalse de Jándula, Embalse de Guadalmena (Margalef et al., 1976); (Avilés et González Ramos, 1981); Laguna Grande; Embalse de Guadalén. MÁLAGA: Embalse de Guadalteba (Margalef et al., 1976). SEVILLA: Embalse de La Minilla (Margalef et al., 1976); Laguna Escalera.

\section{Coelastrum pseudomicroporum Koršikov 1953}

CÁDIZ: Embalse de los Hurones. CÓRDOBA: Laguna de Zóñar. GRANADA: Laguna de Río Seco Inferior. JAÉN: Laguna Grande; Embalse de Guadalén. SEVILLA: Embalse de Cala; Embalse de La Marciaga.

Coelastrum proboscideum Bohlin in Wittrock et Nordstedt 1896

GRANADA: Cartuja (Sánchez Castillo, 1983).

Crucigenia mucronata (G. M. Smith) Komárek 1974

CÁDIZ: Embalse de los Hurones. CÓRDOBA: Embalse de Puente Nuevo.

\section{Crucigenia quadrata Morren 1830}

CÁDIZ: Embalse de Celemín (Margalef et al., 1976). HUELVA: (Avilés et González Ramos, 1981). JAÉN: Embalse de Guadalmena (Margalef et al., 1976). SEVILLA: Embalse de La Minilla (Margalef et al., 1976); Laguna Escalera.

Crucigenia tetrapedia (Kirchner) W. \& G. S. West 1902

CÁDIZ: (Avilés et González Ramos, 1981); Embalse de Guadarranque (Margalef et al., 1976); Embalse de los Hurones. CÓRDOBA: (Avilés et González Ramos, 1981); Laguna de Zóñar. HUELVA: Embalse de La Minilla (Toja et al., 1983); (Avilés et González Ramos, 1981). JAÉN: Embalse de Guadalmena (Margalef et al., 1976); (Avilés et González Ramos, 1981). MÁLAGA: (Avilés et González Ramos, 1981). SEVILLA: Embalse de La
Minilla (Toja, 1980); (Avilés et González Ramos, 1981).

Crucigeniella apiculata (Lemmermann) Komárek 1974

CÁDIZ: Embalse de Celemín (Margalef et al., 1976). CÓRDOBA: Embalse de Iznájar. HUELVA: (Avilés et González Ramos, 1981). JAÉN: Embalse de Guadalén; Embalse de Panzacola; Embalse de Mengíbar. SEVILLA: Embalse de Alcalá del Río.

Crucigeniella pulchra (W. et G. S. West) Komárek 1974

JAÉN: Embalse de Guadalén. SEVILLA: Embalse de Cala.

Crucigeniella rectangularis (Nägeli) Komárek 1974

= Crucigenia rectangularis (Nägeli) Gay 1891

ALMERÍA: Embalse del Almanzora. CÓRDOBA: (Avilés et González Ramos, 1981). HUELVA: (Avilés et González Ramos, 1981); Laguna en Punta Umbría (Doñana) (Margalef, 1976); Embalse de Aracena (Toja et al., 1983). JAÉN: (Avilés et González Ramos, 1981). SEVILLA: Embalse de La Minilla (Toja, 1980); (Avilés et González Ramos, 1981).

Desmodesmus abundans (Kirchner) Hegewald 2000

= Scenedesmus abundans (Kirchner) Chodat

1913 = Scenedesmus quadrispina Chodat 1913

= Scenedesmus sempervirens Chodat 1913

CÓRDOBA: Embalse El Retortillo (Margalef et al., 1976). GRANADA: Laguna Gemela, Majano, Virgen Media (Sierra Nevada) (Sánchez Castillo, 1986, 1988); Laguna Gemela, Majano, Virgen Media, La Caldera (Sierra Nevada) (Sánchez Castillo et al., 1989); Embalse de los Bermejales (Pérez Martínez, 1992). HUELVA: Río Tinto (González Guerrero, 1950); Marisma del Guadalquivir, Laguna Dulce, Laguna Grande (dulce), Acebuche de Matalascañas, Charca del Toro, Laguna de Santa Olalla (Doñana) (Margalef, 1976); Embalse de Aracena (Margalef et al., 1976); Laguna Dulce. JAÉN: Embalse de Jándula (Margalef et al., 1976).

Desmodesmus armatus (Chodat) Hegewald 2000 = Scenedesmus armatus (Chodat) Chodat 
1913

ALMERÍA: Laguna de Garrucha. CÁDIZ: Embalse de los Hurones. CÓRDOBA: (Avilés et González Ramos, 1981); Laguna de Zóñar; Embalse de Iznájar. GRANADA: Embalse de Moraleda, Laguna de Alcantar, Laguna de Charco Negro (de la Rosa, 1992). HUELVA: Acebuche de Matalascañas (Doñana) (Margalef, 1976); (Avilés et González Ramos, 1981). JAÉN: (Avilés et González Ramos, 1981); Embalse de Vadomojón; Embalse de Guadalén. SEVILLA: Laguna Escalera.

\section{Desmodesmus armatus var. bicaudatus} (Guglielmetti) Hegewald 2000

= Scenedesmus armatus var. bicaudatus (Guglielmetti) Chodat $1926=$ Scenedesmus bicaudatus (Hansgirg) Chodat 1926, nom illeg. = Scenedesmus semipulcher Hortobágyi 1960

HUELVA: Embalse de Aracena (Toja et al., 1983). SEVILLA: Embalse de La Minilla (Toja, 1976; Toja, 1980).

Desmodesmus armatus var. spinosus (Fritsch et Rich) Hegewald 2000

= Scenedesmus armatus var. spinosus Fritsch et Rich 1929 = Scenedesmus soli Hortobágyi 1960

= Scenedesmus denticulatus var. costatus Hortobágyi $1960=$ Scenedesmus pseudohystrix Masjuk 1962

GRANADA: Virgen Media, Aguas Verdes, Río Seco superior, Gemela (Sierra Nevada) (Sánchez Castillo et al., 1989); Laguna Virgen Superior, La Caldera, Laguna de Aguas Verdes, Gemela, Río Seco, Virgen Media (Sierra Nevada) (Sánchez Castillo, 1986); Lagunas de Sierra Nevada (Sánchez Castillo, 1988).

Desmodesmus brasiliensis (Bohlin) Hegewald 2000

= Scenedesmus brasiliensis Bohlin 1897

CÓRDOBA: Embalse de Guadalmellato. HUELVA: Embalse de Aracena (Margalef et al., 1976). JAÉN: Embalse de Panzacola.

Desmodesmus communis (Hegewald) Hegewald 2000

= Scenedesmus communis Hegewald $1977=$ Scenedesmus quadricauda (Turpin) Brébisson sensu Chodat 1913, 1926

ALMERÍA: Albuferas de Adra (Carrillo et al.,
1987; Sánchez Castillo, 1987a). CÁDIZ: (Avilés et González Ramos, 1981). CÓRDOBA: (Avilés et González Ramos, 1981). GRANADA: (Cambra et al., 1989); Laguna del Padul (Pérez Martínez et Sánchez Castillo, 1990); Embalse de Moraleda (de la Rosa, 1992). HUELVA: Río Tinto (González Guerrero, 1950); Laguna Dulce, Lagunita de las Dunas, Laguna Grande (dulce), El Rocío, Laguna de Santa Olalla (Doñana) (Margalef, 1976); Embalse de Aracena (Margalef et al., 1976; Toja et al., 1983); (Avilés et González Ramos, 1981); Laguna Dulce. JAÉN: Embalse de Jándula, Embalse de Guadalmena (Margalef et al., 1976); (Avilés et González Ramos, 1981); Embalse de Vadomojón; Laguna Grande; Embalse de Guadalén. MÁLAGA: (Avilés et González Ramos, 1981). SEVILLA: Embalse de Torre del Águila (Margalef et al., 1976); (Avilés et González Ramos, 1981); Embalse de La Minilla (Toja, 1980; Casco et Toja, 1991, 1994); Laguna Escalera.

Desmodesmus communis var. rectangularis (G. S. West) Hegewald 2000

= Scenedesmus quadricauda var. rectangularis

G. S. West $1914=$ Scenedesmus smithii Chodat 1926

CÓRDOBA: (Avilés et González Ramos, 1981).

Desmodesmus costato-granulatus (Skuja) Hegewald 2000

= Scenedesmus costato-granulatus Skuja 1948 = Scenedesmus granulatus W. \& G. S. West sensu auct. post. $=$ Didymocystis inconspicua Koršikov 1953 = Didymocystis lineata Koršikov 1953

GRANADA: Embalse de los Bermejales. CÓRDOBA: Embalse de Iznájar. HUELVA: Doñana (Margalef, 1976).

Desmodesmus denticulatus (Lagerheim) An, Friedl et Hegewald 1999

= Scenedesmus denticulatus Lagerheim 1882

HUELVA: Charco del Toro, Marisma de Hinojos (Doñana) (Margalef, 1976). JAÉN: (González Guerrero, 1928).

Desmodesmus denticulatus var. linearis (Hansgirg) Hegewald 2000

= Scenedesmus denticulatus var. linearis Hansgirg 1888 = Scenedesmus brevispina (G. M. 
Smith) Chodat 1926 = Scenedesmus longus var. brevispina G. M. Smith 1916

CÁDIZ: La Cortadura (González Guerrero, 1946).

Desmodesmus cf. dispar (Brébisson) Hegewald 2000

= Scenedesmus dispar Brébisson 1856

ALMERÍA: Laguna de Garrucha.

Desmodesmus grahneisii (Heynig) Hegewald 2000

= Didymocystis grahneisii Heynig 1962

GRANADA: Embalse de Canales.

Desmodesmus hystrix (Lagerheim) Hegewald 2000

= Scenedesmus hystrix Lagerheim 1882

CÓRDOBA: (Avilés et González Ramos, 1981). HUELVA: (Avilés et González Ramos, 1981). JAÉN: (Avilés et González Ramos, 1981). MÁlAGA: (Avilés et González Ramos, 1981).

SEVILLA: (Avilés et González Ramos, 1981).

Desmodesmus intermedius (Chodat) Hegewald 2000

= Scenedesmus intermedius Chodat 1926

CÁDIZ: (Avilés et González Ramos, 1981).

GRANADA: Diputación (Sánchez Castillo, 1983).

Desmodesmus intermedius var. acutispinus (Roll) Hegewald 2000

= Scenedesmus bicaudatus Dedussenko 1925 MÁLAGA: (Margalef, 1944).

Desmodesmus maximus (W. et G. S. West) Hegewald 2000

= Scenedesmus quadricauda var. maximus $\mathrm{W}$. et G. S. West $1895=$ Scenedesmus maximus (W. et G. S. West) Chodat 1913

HUELVA: Río Tinto (González Guerrero, 1950); Laguna Dulce, Lagunita de las Dunas, Charca del Toro (Doñana) (Margalef, 1976).

Desmodesmus opoliensis (Richter) Hegewald 2000

= Scenedesmus opoliensis Richter 1896

CÁDIZ: Embalse de Bornos (Margalef et al., 1976). HUELVA: Laguna Dulce, Laguna de Santa Olalla, Charco del Toro (Doñana) (Margalef, 1976);
Laguna Dulce. JAÉN: Embalse de Guadalmena (Margalef et al., 1976). SEVILLA: Embalse de Torre del Águila (Margalef et al., 1976); Embalse de Alcalá del Río; Embalse de Cala; Embalse de La Marciaga.

Desmodesmus opoliensis var. opoliensis (Richter) Hegewald 2000

= Scenedesmus opoliensis var. opoliensis Richter 1896

HUELVA: Laguna Dulce; Laguna de Santa Olalla.

Desmodesmus opoliensis var. carinatus (Lemmermann) Hegewald 2000

= Scenedesmus opoliensis var. carinatus Lemmermann 1899

ALMERÍA: Laguna de Garrucha.

Desmodesmus opoliensis var. mononensis (Chodat) Hegewald 2000

= Scenedesmus opoliensis var. mononensis Chodat 1926

HUELVA: Laguna de Santa Olalla. JAÉN: Laguna Grande.

Desmodesmus protuberans (Fritsch et Rich) Hegewald 2000

= Scenedesmus protuberans Fritsch et Rich 1929

HUELVA: Laguna de Sopetón.

Desmodesmus spinosus (Chodat) Hegewald 2000 = Scenedesmus spinosus Chodat $1913=$ Scenedesmus corallinus Chodat 1926= Scenedesmus breviaculeatus Chodat 1926

GRANADA: Laguna de la Virgen Superior, Lagunas de Sierra Nevada (Sánchez Castillo, 1986, 1988). HUELVA: Laguna de Santa Olalla.

Desmodesmus subspicatus (Chodat) Hegewald 2000

= Scenedesmus subspicatus Chodat 1926

GRANADA: Parque del Triunfo (Sánchez Castillo, 1980, 1983). HUELVA: Laguna de Sopetón.

Hariotina polychorda (Koršikov) Hegewald in Hegewald et al. 2002

= Coelastrum reticulatum var. polychordum 
Koršikov $1953=$ Coelastrum polychordum (Koršikov) Hindák 1977

CÁDIZ: Embalse de los Hurones.

\section{Hariotina reticulata Dangeard 1889}

$=$ Coelastrum reticulatum (Dangeard) Senn 1899

CÁDIZ: Embalse de los Hurones. CÓRDOBA: Embalse El Retortillo (Margalef et al., 1976); (Avilés et González Ramos, 1981); Embalse de Iznájar; Embalse de Puente Nuevo; Embalse de Guadalmellato. GRANADA: Facultad de Filosofía y Letras (Sánchez Castillo, 1983); Embalse de Cubillas. HUELVA: (Avilés et González Ramos, 1981). JAÉN: Embalse de Rúmblar (Margalef et al., 1976); (Avilés et González Ramos, 1981); Embalse de Rúmblar; Embalse de Panzacola; Embalse de Guadalén. SEVILLA: Embalse del Pintado, Embalse de La Minilla (Margalef et al., 1976; Toja, 1980; Toja et al., 1983); Embalse de Alcalá del Río.

Scenedesmus acuminatus (Lagerheim) Chodat 1902 s. 1.

= Scenedesmus falcatus Chodat 1894, nom illeg.

ALMERÍA: Laguna Nueva (Albufera de Adra) (Carrillo et al., 1987). CÓRDOBA: (Avilés et González Ramos, 1981). HUELVA: Laguna del Brezo, Laguna Dulce, Lagunita en las Dunas, Laguna Grande, Marisma de Hinojos, Laguna de Santa Olalla, Charca del Toro (Doñana) (Margalef, 1976); (Avilés et González Ramos, 1981). JAÉN: Embalse de Jándula (Margalef et al., 1976); Embalse de Guadalén. MÁLAGA: Embalse de Guadalteba (Margalef et al., 1976). SEVILLA: Embalse de Torre del Águila (Margalef et al., 1976); (Avilés et González Ramos, 1981); Embalse de La Minilla (Toja, 1980; Casco et Toja, 1994); Embalse de Alcalá del Río; Embalse de La Marciaga; Laguna Escalera.

\section{Scenedesmus arcuatus var. arcuatus}

(Lemmermann) Lemmermann 1899

CÁDIZ: Embalse de Bornos (Margalef et al., 1976); Embalse de los Hurones. CÓRDOBA: Embalse El Retortillo (Margalef et al., 1976); Laguna de Zóñar; Embalse de Puente Nuevo; Embalse de Iznájar. GRANADA: Embalse de Cubillas. HUELVA: Embalse de Aracena (Margalef et al., 1976). JAÉN: Embalse de Vadomojón; Embalse de Guadalén.
MÁLAGA: Embalse de Guadalteba (Margalef et al., 1976). SEVILLA: Laguna Escalera.

Scenedesmus arcuatus var. platydiscus G. M. Smith 1916

CÁDIZ : Embalse de los Hurones. GRANADA: Laguna de Río Seco Inferior; Laguna de Aguas Verdes. JAÉN: Embalse de Guadalén; Laguna Grande. MÁLAGA: Laguna Grande de Archidona. SEVILLA: Laguna Escalera; Embalse de Alcalá del Río; Embalse de La Marciaga.

Scenedesmus ecornis (Ehrenberg ex Ralfs) Chodat 1926

ALMERÍA: Laguna Nueva (Albufera de Adra) (Carrillo et al., 1987; Sánchez Castillo, 1987a). CÁDIZ: Fuente en el Parque del Genovés (González Guerrero, 1947) (sub Scenedesmus bijuga); Embalse de Bornos, Embalse de Guadarranque, Embalse de Celemín (Margalef et al., 1976); CÓRDOBA: Embalse de Guadanuño (Margalef et al., 1976). GRANADA: Laguna de La Caldera (Martínez Silvestre, 1977); Charcos turberales, $7^{\text {a }}$ Laguna (Mulhacén-Alcazaba, Sierra Nevada) (González Guerrero, 1981) (sub Scenedesmus bijuga); Albaicín (Sánchez Castillo, 1983); Laguna del Padul (Pérez Martínez et Sánchez Castillo, 1990); Embalse de los Bermejales (Pérez Martínez, 1992). HUELVA: Laguna Dulce, Marisma del Guadalquivir, Laguna de Santa Olalla, Laguna de Punta Umbría (Doñana) (Margalef, 1976); Embalse de Aracena (Margalef et al., 1976). JAÉN: (Aboal, 1996). MÁLAGA: Embalse de Guadalhorce, Embalse de Guadalteba (Margalef et al., 1976). SEVILLA: Embalse de Torre del Águila, Embalse de La Minilla (Margalef et al., 1976); Embalse de La Minilla (Casco et Toja, 1994).

\section{Scenedesmus ellipticus Corda 1835}

= Scenedesmus linearis Komárek 1974

CÁDIZ: (Avilés et González Ramos, 1981). CÓRDOBA: (Avilés et González Ramos, 1981); Embalse de Iznájar; Laguna de Zóñar. HUELVA: (Avilés et González Ramos, 1981). JAÉN: (Avilés et González Ramos, 1981); Embalse de Vadomojón; Laguna Grande; Embalse de Mengíbar. SEVILLA: (González Fragoso, 1893); Embalse de Alcalá del Río.

Scenedesmus incrassatulus Bohlin 1897

SEVILLA: Embalse de La Minilla (Toja, 1980; 
Toja et al., 1983).

Scenedesmus obliquus (Turpin) Kützing 1833

= Scenedesmus acutus Meyen $1829=$

Scenedesmus dimorphus (Turpin) Kützing 1833

CÁDIZ: Balneario de Fuente Amarga

(González Guerrero, 1946). GRANADA: Embalse de Moraleda, Laguna de Charco Negro (de la Rosa, 1992); Embalse de los Bermejales (Pérez Martínez, 1992). HUELVA: Río Tinto (González Guerrero, 1950); Marisma de Hinojos (Doñana) (Margalef, 1976). JAÉN: Embalse de Jándula (Margalef et al., 1976). MÁLAGA: (Margalef, 1944). SEVILLA: Embalse de Cala, Embalse de Torre del Águila (Margalef et al., 1976); Embalse de La Minilla (Toja, 1980).

Scenedesmus obliquus var. dimorphus (Turpin) Hansgirg 1888

ALMERÍA: Laguna de Garrucha. CÓRDOBA: Laguna de Zóñar. GRANADA: Embalse de Moraleda (de la Rosa, 1992); Embalse de Béznar. HUELVA: Marisma de Hinojos (Doñana) (Margalef, 1976). JAÉN: Embalse de Vadomojón. SEVILLA: Laguna Escalera; Embalse de La Marciaga.

\section{Scenedesmus obtusus Meyen 1829}

$=$ Scenedesmus alternans Reinsch $1867=$ Scenedesmus bijuga var. alternans (Reinsch) Borge 1906

ALMERÍA: Laguna de Mojácar; Balsa de Punta Entinas. CÁDIZ: La Cortadura (González Guerrero, 1946). JAÉN: (González Guerrero, 1928).

Scenedesmus raciborskii Woloszynska 1914

CÁDIZ: Embalse de los Hurones. JAÉN: Embalse de Guadalén. MÁLAGA: Laguna Grande de Archidona.

\section{Scenedesmus verrucosus Roll 1925}

= Scenedesmus ecornis var. disciformis (Chodat) Chodat $1926=$ Scenedesmus disciformis (Chodat) Fott et Komárek 1960

CÁDIZ: (Avilés et González Ramos, 1981). CÓRDOBA: (Avilés et González Ramos, 1981). GRANADA: (Avilés et González Ramos, 1981). HUELVA: Laguna Dulce (Doñana) (Margalef, 1976); (Avilés et González Ramos, 1981). JAÉN: (Avilés et González Ramos, 1981). MÁLAGA: (Avilés et González Ramos, 1981). SEVILLA:
Embalse El Gergal (Toja et al., 1983); (Avilés et González Ramos, 1981).

Tetradesmus wisconsinensis G. M. Smith 1913 HUELVA: Laguna de Santa Olalla, Charco del Toro (Margalef, 1976).

\section{Tetrastrum komarekii Hindák 1977}

JAÉN: Embalse de Panzacola.

Tetrastrum staurogeniaeforme (Schröder) Lemmermann 1900

CÁDIZ: (Avilés et González Ramos, 1981). CÓRDOBA: Embalse de Puente Nuevo. JAÉN: (Avilés et González Ramos, 1981). SEVILLA: Embalse de Alcalá del Río.

Tetrastrum triangulare (Chodat) Komárek 1974 CÁDIZ: Fuente Amarga (González Guerrero, 1946); Embalse de los Hurones. CÓRDOBA: Embalse de Iznájar. GRANADA: Embalse de los Bermejales (Pérez Martínez, 1992). JAÉN: Embalse de Mengíbar. SEVILLA: Embalse de Alcalá del Río.

Westella botryoides (W. West) De-Wildermann 1897

JAÉN: Embalse de Guadalmena, Embalse de Guadalén, Embalse de Tranco de Beas (Margalef et al., 1976); Embalse de Guadalén; Embalse de Mengíbar.

\section{Nuevas citas}

En total, en las muestras tomadas a lo largo de este trabajo, hemos hallado 91 especies de algas verdes cocales. Algunas de estas especies se presentan en diferentes variedades. De todas ellas, 9 no habían sido citadas anteriormente en la Península Ibérica: Botryococcus terribilis, Desmodesmus grahneisii, Dicellula geminata, Dichotomococcus curvatus, Dictyosphaerium elongatum, Franceia ovalis, Paradoxia multiseta, Tetrachlorella ornata, Chlorella minutissima.

Además, otras tres especies sólo habían sido citadas en Portugal, por lo que se citan por primera vez en España: Kirchneriella rotunda, Crucigenia mucronata, Treubaria schmidlei. 
Entre las especies ya citadas en España, en este trabajo se aportan 27 nuevas citas para Andalucía: Ankistrodesmus stipitatus, Ankyra lanceolata, Closteriopsis acicularis, Coelastrum astroideum, Coelastrum indicum, Coelastrum pseudomicroporum, Coenocystis tapasteana, Crucigeniella pulchra, Desmodesmus costato-granulatus, Desmodesmus cf. dispar, Dictyosphaerium tetrachotomum, Gloeotaenium loitlesbergerianum, Hariotina polychorda, Kirchneriella contorta, Kirchneriella irregularis, Kirchneriella subcapitata, Lobocystis cf. planctonica, Micractinium crassisetum, Monoraphidium arcuatum, Oocystis submarina, Pseudoschroederia antillarum, Pseudoschroederia robusta, Quadricoccus ellipticus, Scenedesmus raciborskii, Tetrachlorlla alternans, Tetraedron regulare, Tetrastrum komarekii.

Cabe destacar que en el área de estudio han sido localizadas algunas especies de distribución preferentemente tropical: Coenocystis tapasteana, Coelastrum indicum, Botryococcus terribilis, Crucigenia mucronata, Pediastrum boryanum var. brevicorne y Hariotina polychorda.

\section{BIBLIOGRAFÍA}

ABOAL, M. -1988- Aportación al conocimiento de las algas epicontinentales del S.E de España VII. Clorofíceas (Chlorophyceae Wille in Warming, 1884). Candoella 43: 521-548.

ARMENGOL, J., J. CATALÁN, N. GABELLONE, D. JAUME, J. de MANUEL, E. MARTÍ, J. A. MORGUÍ, J. NOLLA, J. PEÑUELAS, M. REAL, J. L. RIERA, S. SABATER, F. SABATER \& J. TOJA -1990- A comparative limnological study of the Guadalhorce reservoirs system (Málaga, S.E. Spain). Scientia Gerundensis 16(2): 27-41.

BAREA ARCO, J., C. PÉREZ MARTÍNEZ \& R. MORALES BAQUERO -2001- Evidence of a mutualistic relationship between an algal epibiont and its host, Daphnia pulicaria. Limnol. Oceanogr. 46(4): 871-881.
BOLÍVAR GALIANO, F. -1994- Diagnosis y tratamiento del deterioro por microalgas en los Palacios Nazaries de La Alhambra. Tesis doctoral. Universidad de Granada, Granada. $246 \mathrm{pp}$.

BUDDE, H. -1929- Beitrag zur Algenflora der fliessenden Gewasser Spaniens. Arch. Hydrobiol. 20: 427-470.

CAMBRA, J., J. NOLLA y S. SABATER -1989Composición fitoplanctónica en embalses de pequeño volumen del este de la Península Ibérica. Bol. Soc. Broteriana 62:5-18.

CAMBRA SÁNCHEZ, J., M. ÁLVAREZ COBELAS y M. ABOAL SANJURJO -1998-Lista florística y bibliográfica de los clorófitos (Chlorophyta) de la Península Ibérica, Islas Baleares e Islas Canarias. García Avilés, J. \& Rico, E., Ed. Listas de la flora y fauna de las aguas continentales de la Península Ibérica, 14, Burgos. 614 pp.

CARRILLO, P., L. CRUZ PIZARRO, R. MORALES y P. M. SÁNCHEZ CASTILLO -1987- Cambios estacionales en las comunidades de fitoplancton y de zooplancton de la albufera de Adra. Limnetica 3(2): 243-254.

CARRILlO, P., L. CRUZ PIZARRO y P. M. SÁNCHEZ CASTILLO -1990- Analysis of phytoplankton-zooplankton relationships in an oligotrophic lake under natural and manipulated conditions. Hydrobiologia 200/201: 49-58.

CASCO, M. A. -1990- El perifiton del embalse de La Minilla: Relaciones con el fitoplancton y contribución a la producción total. Tesis doctoral. Universidad de Sevilla, Sevilla. 373 pp.

CASCO, M. A. \& J. TOJA-1991- Benthic microalgae of La Minilla Reservoir (South West Spain). Verh. Internat. Verein. Limnol. 24: 1386-1389.

CASCO, M. A. \& J. TOJA -1994- The distribution and interaction of algal communities in reservoirs. Arch. Hydrobiol. Beih. 40: 85-96.

CIRUJANO, S., J. CAMBRA, P. M. SÁNCHEZ CASTILLO, A. MECO y N. FLOR ARNAU -2008- Flora Ibérica. Algas Continentales. Carófitos (Characeae). Real Jardín Botánico, Madrid. 132 pp.

COMAS GONZÁLEZ,A. -1996- Las Chlorococcales dulciacuícolas de Cuba. Kies, L. \& Schnetter, R., Eds. Bibliotheca Phycologica, 99, J. Cramer, Stuttgart. 192 pp.

COMAS GONZÁLEZ, A., M. C. PÉREZ BALIERO \& J. GONZÁLEZ DEL RÍO RAMS -2006- 
Pediastrum willei nom. et sp. nov. (Chlorophyta, Neochloridales) from the Ebro river, Spain and its relations to P. muticum Kütz. sensu Brunnthaler 1915 pro parte. Algol. Stud. 120: 5-13.

DE LA ROSA, J. C. -1992-Estudio del fitoplancton de varias lagunas de las provincias de Granada y Málaga. Memoria de licenciatura. Universidad de Granada, Granada. 215 pp.

DELGADO MOLINA, J. A., P. CARRILLO, J. M. MEDINA SÁNCHEZ, M. VILLAR ARGAIZ \& F. J. BULLEJOS -2009- Interactive effects of phosphorus loads and ambient ultraviolet radiation on the algal community in a highmountain lake. J. Plankton Res. 31(6): 619634.

FANÉS TREVIÑO, I. -2008- Estudios taxonómicos en algas verdes cocales del sur de España. Tesis doctoral. Universidad de Granada, Granada. $325 \mathrm{pp}$.

FANÉS TREVIÑO, I., P. SÁNCHEZ CASTILLO \& A. COMAS GONZÁLEZ -2009Contribution to the taxonomic study of the family Botryococcaceae (Trebouxiophyceae, chlorophyta) in southern Spain. Cryptogamie, Algologie 30(1): 17-30.

GARCÍA, C. M., R. GARCÍA RUIZ, M. RENDÓN, F. X. NIELL \& J. LUCENA-1997- Hydrological cycle and interannual variability of the aquatic community in a temporary saline lake (Fuente de Piedra, Southern Spain). Hydrobiologia 345: 131-141.

GARCÍA RUIZ, R., C. M. GARCÍA y F. X. NIELL -1993- El sistema acuático del sistema de Fuente de Piedra después de una pluviosidad anormalmente alta (1989-90). Actas VI Congr. Esp. Limnología: 75-82.

GONZÁLEZ GUERRERO, P. -1946- Cianofíceas y algas continentales de Cádiz. An. J. Bot. Madrid 6(1): 237-335.

GONZÁLEZ GUERRERO, P. -1947- Algas invernales gaditanas. An. J. Bot. Madrid 7: 433-456.

GONZÁLEZ GUERRERO, P. -1950- Algas del río Tinto. An. J. Bot. Madrid 9: 111-128.

GONZÁLEZ GUERRERO, P. -1981- Algas de la orotropia elevada española (cerro del Mulhacén). Sierra Nevada en Granada (España). Inédito.

HINDÁK, F. -1977- Studies on the chlorococcal algae (Chlorophyceae). I. Biologické Pracé 23, VEDA, Bratislava. $190 \mathrm{pp}$.
HINDÁK, F. -1980- Studies on the chlorococcal algae (Chlorophyceae). II. Biologické Pracé 26, VEDA, Bratislava.

HINDÁK, F. -1984- Studies on the chlorococcal algae (Chlorophyceae). III. Biologické Pracé, 30, VEDA, Bratislava. 308 pp.

HINDÁK, F. -1988- Studies on the chlorococcal algae (Chlorophyceae). IV. Biologické Pracé, 34, VEDA, Bratislava. 263 pp.

HINDÁK, F. -1990- Studies on the chlorococcal algae (Chlorophyceae). V. Biologické Pracé, VEDA, Bratislava. 225 pp.

HOYOS, C. de -1996- Limnología del lago de Sanabria: variabilidad interanual del fitoplancton. Tesis doctoral. Universidad de Salamanca. 438 pp.

KOMÁREK, J. \& B. FOTT -1983- Chlorophyceae (Grünalgen). Ordnung: Chlorococcales. HuberPestalozzi, G., Ed. Das Phytoplankton des Süßwassers. Systematik und Biologie, 7, Schweizerwart'ssche Verlagbuchhandlung, Stuttgart. 1044 pp.

KOMÁREK, J. \& V. JANKOVSKÁ -2001- Review of the green algal genus Pediastrum; implication for pollen-analytical research. Kies, L. \& Schnetter, R., Eds. Bibliotheca Phycologica, 108. J. Cramer, Stuttgart. 127 pp.

KOMÁREK, J. \& P. MARVAN, P. -1992Morphological differences in natural populations of the genus Botryococcus (Chlorophyceae). Arch. Protistenk. 141: 65-100.

KOMÁREK, J. \& J. PERMAN -1978- Review of the genus Dictyosphaerium (Chlorococcales). Arch. Hydrobiol. Suppl. 51, Algol. Stud. 18: 33-45.

KOMÁRKOVÁ-LEGNEROVÁ, J. -1969- The systematics and ontogenesis of the genera Ankistrodesmus Corda and Monoraphidium gen. nov. In Studies in Phycology (Fott, B., ed.), pp. 75-122, Praha.

LINARES CUESTA, E. -2003- Las diatomeas bentónicas de las lagunas del Parque Nacional de Sierra Nevada. Estudio comparado con las colecciones del Herbario de la Universidad de Granada (GDA). Tesis doctoral. Universidad de Granada, Granada. 327 pp.

MARGALEF, R. -1944- Datos para la flora algológica de nuestras aguas dulces. Publ. Inst. Bot. Barna. 4.

MARGALEF, R. -1976- Algas de agua dulce de Doñana. Oecol. Aquat. 2: 79-93. 
MARGALEF, R., D. PLANAS, J. ARMENGOL, A. VIDAL, N. PRAT, A. GUISET, J. TOJA y M. ESTRADA -1976- Limnología de los embalses españoles. MOPU. Madrid. 422 pp.

MARTÍNEZ SILVESTRE, R. -1977- Phytoplankton species, biomass and diversity in lake La Caldera (Spain). Acta Hydrobiologica 19(2): 95-107.

MORALES BAQUERO, R., P. CARRILLO, L. CRUZ PIZARRO \& P. SÁNCHEZ CASTILLO -1992- Southernmost high mountain lakes in Europe (Sierra Nevada) as reference sites for pollution and climate change monitoring. Limnetica 8: 39-47.

NEGRO, A. I. -2005- Ecología del fitoplancton de lagunas y turberas de las sierras Segundera y Cabrera y de la Cordillera Cantábrica. Tesis doctoral. Universidad de Salamanca, Salamanca.

PERAZA ZURITA, Y., G. CULTRONE, P. M. SÁNCHEZ CASTILLO \& F. BOLÍVAR GALIANO -2002- Il biodeterioramento delle fontane dei Reales Alcázares di Siviglia e dell'Alhambra di Granada (Spagna). Science and Technology for Cultural Heritage 11(1-2): 111-118.

PÉREZ BALIERO, M. C., A. COMAS, J. G. DEL RÍO \& J. P. SIERRA -2002- Planktonic Chlorophyceae from the lower Ebro River (Spain). Acta Bot. Croat. 61(2): 99-124.

PÉREZ MARTÍNEZ, C. -1992-Respuesta del fitoplancton a la manipulación experimental conjunta de la presión de depredación y de la disponibilidad de nutrientes en un embalse de moderada eutrofia. Tesis doctoral. Facultad de Ciencias, Universidad de Granada, Granada. $168 \mathrm{pp}$.

PÉREZ MARTÍNEZ, C. \& L. CRUZ PIZARRO 1995- Species-specific phytoplankton responses to nutrients and zooplankton manipulations in enclosure experiments. Freshw. Biol. 33: 193-203.

PÉREZ MARTÍNEZ, C. \& P. M. SÁNCHEZ CASTILLO -1990- Dinámica de la comunidad fitoplanctónica de una laguna somera (Padul, Granada). Scientia Gerundensis 16(2): 99-112. PÉREZ MARTÍNEZ, C., J. BAREA ARCO \& P. SÁNCHEZ CASTILLO -2001- Dispersal and colonization of the epibiont alga Korshikoviella gracilipes (Chlorophyceae) on Daphnia pulicaria (Cladocera). J. Phycol. 37(5): 724-730.
PÉREZ MARTÍNEZ, C., J. BAREA ARCO, J. M. CONDE PORCUNA \& R. MORALES BAQUERO -2007- Reproduction strategies of Daphnia pulicaria population in a high mountain lake of Southern Spain. Hydrobiologia 594(1): 75-82.

PHILIPOSE, M. T. -1967- Chlorococcales. Indian Council of Agricultural Research, New Delhi. 365 pp.

REHÁKOVÁ, H. -1969- Die Variabilität der Arten der Gattung Oocystis A. Braun. In Studies in Phycology (Fott, B., ed.), pp. 145-198, Praha.

RIVERA GONZÁLEZ, M. C. -2004- Estudio taxonómico de las diatomeas planctónicas del litoral de Andalucía. Caracterización de las comunidades potencialmente tóxicas o nocivas. Tesis doctoral. Universidad de Granada, Granada.

ROJO, C. -1990- Estructura de la comunidad fitoplanctónica de la laguna hipertrófica " $E l$ Porcal" (Madrid). Tesis doctoral. Universidad de Valencia, Valencia. 343 pp.

ROMO, S. G. -1991- Estudio del fitoplancton de la Albufera de Valencia, una laguna hipertrófica y somera, entre 1980 y 1988. Tesis doctoral. Universidad de Valencia, Valencia. 197 pp.

SABATER, S. \& J. NOLLA -1991- Distributional patterns of phytoplankton in Spanish reservoirs: First results and comparison alter fifteen years. Verh. Int. Verein. Limnol. 24: 1371-1375.

SÁNCHEZ CASTILLO, P. M. -1980- Algas verdes $y$ azules de los estanques y fuentes de Granada. Tesina de Licenciatura. Facultad de Ciencias. Universidad de Granada.

SÁNCHEZ CASTILLO, P. M. -1982- Estudio limnológico de dos ecosistemas hídricos de la provincia de Almería. Instituto de Estudios Almerienses, Diputación de Almería, inédito.

SÁNCHEZ CASTILLO, P. M. -1983-Clorofitas de la ciudad de Granada. Trabajos del Departamento de Botánica de la Universidad de Granada 3: 63-79.

SÁNCHEZ CASTILLO, P. M. -1986- Estudio de las comunidades fitoplanctónicas de las lagunas de alta montaña de Sierra Nevada. Tesis doctoral. Universidad de Granada, Granada. 246 pp.

SÁNCHEZ CASTILLO, P. M. -1987a- Influencia de la salinidad sobre las poblaciones algales de tres lagunas litorales (Albuferas de Adra, Almería). Limnetica 3: 47-53. 
SÁNCHEZ CASTILLO, P. M. -1987b- Estudio del ciclo biológico de Korshikoviella gracilipes (Lambert) Silva (Chlorococcales, Chlorophyta). Phycologia 26(4): 496-500.

SÁNCHEZ CASTILLO, P. M. -1988- Algas de las lagunas de alta montaña de Sierra Nevada (Granada, España). Acta Bot. Malacitana 13: 21-52.

SÁNCHEZ CASTILLO, P. M. y J. A. GIL -1982Vegetación criptogámica de las tobas de la provincia de Granada (España). Coll. Bot. 13(1): 231-245.

SÁNCHEZ CASTILLO, P. M., L. CRUZ PIZARRO \& P. CARRILLO -1989- Caracterización del fitoplancton de las lagunas de alta montaña de Sierra Nevada (Granada, España) en relación con las características físico-químicas del medio. Limnetica 5: 37-50.

TOJA, J. -1976- Limnología comparada de dos embalses con distinto grado de eutrofia (Aracena y La Minilla). Tesis doctoral. Universidad de Barcelona, Barcelona.

TOJA, J. -1980- Limnología del embalse de La Minilla durante 1976. I. Ciclo del fitoplancton en relación con los factores del medio. Oecol. Aquat. 4: 71-88.

TOJA, J. \& M. A. CASCO -1991- Contribution of phytoplankton and periphyton to the production in a reservoir of S.W. Spain. Oecol. Aquat. 10: 61-76.

TOJA, J., T. LÓPEZ \& N. GABELLONE -1991Successional changes in two dune ponds (Doñana Nacional Park). Verh. Internat. Verein. Limnol. 24: 1556-1559.

TOJA, J., J. GONZÁLEZ RULL \& D. RAMOS 1983- Phytoplankton succession in Aracena, La Minilla and El Gergal reservoirs (Huelva-Sevilla, Spain). Wat. Supply 1(1): 103-113. 